J. Lake Sci. (湖泊科学) , 2015, 27(5):911-916

DOI $10.18307 / 2015.0518$

(C) 2015 by Journal of Lake Sciences

\title{
沉积物再悬浮对食浮游动物鱼类捕食浮游动物的影响”
}

\author{
周礼斌 ${ }^{1,2}$, 陈非洲 ${ }^{1 * *}$ \\ ( 1 : 中国科学院南京地理与湖泊研究所湖泊与环境国家重点实验室, 南京 210008) \\ (2: 中国科学院大学, 北京 100049)
}

摘 要: 在亚热带浅水湖泊中, 由风浪引起沉积物再悬浮是其重要特征之一. 沉积物再悬浮一方面直接影响浮游动物群 落结构, 另一方面通过影响食浮游动物鱼类对浮游动物的捕食而产生间接影响. 以食浮游动物鱼类一一餐条 (Hemiculter leucisculus) 为实验对象, 通过模拟实验, 比较不同再悬浮条件下餐条对浮游动物群落结构的影响. 结果显示, 在有餐条的 处理组中, 大型枝角类短针溞 (Daphnia obtusa) 只在弱再悬浮条件下出现, 并成为枝角类群落的优势种; 在无餐条的处理 组中, 短钝泽生物量随再悬浮强度减弱而增加. 强再悬浮 (无餐条) 和无再悬浮 (有餐条) 条件下, 小型枝角类角突网纹溞 (Ceriodaphnia cornuta) 和盘肠溞 (Chydorus sp.) 为优势种. 无论有、无餐条条件下, 桡足类优势种均为中剑水蚤 (Mesocyclops sp. ), 其生物量随再悬浮强度减弱而增加. 所有处理组中, 轮虫丰度均低于枝角类和桡足类丰度. 研究表明, 在亚热带浅水 湖泊中, 适当的悬浮质浓度既能干扰食浮游动物鱼类对大型浮游动物的捕食, 又不影响大型浮游动物的生存, 使得它们 保持一定的丰度, 通过对浮游植物的摄食, 降低叶绿素 a 浓度.

关键词: 沉积物再悬浮; 浮游动物; 餐条; 群落结构;亚热带浅水湖泊;太湖

\section{Effect of sediment resuspension on predation of planktivorous fish on zooplankton}

\section{ZHOU Libin $^{1,2}$ \& CHEN Feizhou ${ }^{1}$}

(1: State Key Laboratory of Lake Science and Environment, Nanjing Institute of Geography and Limnology, Chinese Academy of Sciences, Nanjing 210008, P. R. China)

(2: University of Chinese Academy of Sciences, Beijing 100049, P. R. China)

Abstract: Sediment resuspension resulted from wave actions is one of the major characters of subtropical shallow lakes. Resuspension can directly alter the zooplankton community structures, and indirectly influence the predation of planktivorous fishes. Our study aimed to reveal how planktivorous fish Hemiculter leucisculus affected the zooplankton community structures in different levels of sediment resuspension. The results showed that in the condition of $H$. leucisculus, large-sized cladoceran Daphnia obtusa was only found and became dominant in the treatment of weak resuspension. Biomass of D. obtusa decreased with the increase of resuspension intensity in treatments without $H$. leucisculus and Chydorus sp. . The Ceriodaphnia cornuta dominated the cladoceran communities in the treatments of strong resuspension without $H$. leucisculus or no resuspension with H. leucisculus. Regardless of fish and resuspension intensity, Mesocyclops sp. was dominant copepod and its biomass increased with the decrease of the resupension intensity. Rotifer abundances were lower than cladoceran and copepod in all treatments. This study indicates that in subtropical shallow lakes, a certain level of sediment resuspension can weaken both the predation of planktivorous fishes and resuspension effect on large-sized cladoceran, which can decrease the chlorophyll-a concentration through increasing the feeding of large-sized cladoceran on algae.

Keywords: Sediment resuspension; zooplankton; Hemiculter leucisculus; community structures; subtropical shallow lakes; Lake Taihu

经典的生物操纵是通过减少或去除食浮游动物鱼类, 增加大型枝角类 (主要是枝角类溞 Daphnia spp.)

* 国家自然科学基金项目(41271523) 和江苏省科技支撑项目 (BE2011820) 联合资助. 2014-10-25 收稿; 201502-25收修改稿. 周礼斌(1989～), 男, 硕士研究生;E-mail : zlbxingxing@ 163.com.

** 通信作者;E-mail:feizhch@ niglas. ac. cn. 
的数量, 从而抑制浮游植物的数量达到改善水质的效果 ${ }^{[1]}$. 此方法已在欧美一些小型湖泊的修复中得到广 泛的应用 ${ }^{[1-2]}$. 与欧美小型湖泊不同的是, 亚热带大型浅水湖泊易受风浪影响造成沉积物再悬浮 ${ }^{[3]}$. 沉积物 再悬浮对水生生态系统有重要的影响, 如降低水体透明度、促进营养盐释放等 ${ }^{[4]}$. 沉积物再悬浮也会改变浮 游植物群落的结构组成, 提高其生物量 ${ }^{[5]}$. 高浓度的悬浮物使大型浮游动物向小型浮游动物转变 ${ }^{[6]}$, 悬浮物 一方面直接妨碍浮游动物的摄食和对食物的吸收 ${ }^{[7-8]}$, 另一方面通过影响浮游植物群落的组成和种群数量 从而影响浮游动物的摄食 ${ }^{[9-10]}$, 高浓度悬浮物也会影响食浮游动物鱼类对浮游动物的捕食 ${ }^{[11]}$, 从而间接影 响浮游动物群落结构. 基于沉积物再悬浮的重要特征, 在易受风浪影响的浅水湖泊中经典生物操纵方法是 否可行仍无法判断,而沉积物再悬浮对上述两种作用的影响程度还需要进一步研究.

鱼类捕食是影响浮游动物种群数量和群落结构的重要因素, 沉积物再悬浮对食浮游动物鱼类捕食的影 响与鱼的种类和功能有关 ${ }^{[12-13]}$, 其中悬浮物对鱼类捕食的影响包括破坏鳃结构和干扰捕食行为等 ${ }^{[14-15]}$. Shoup 等 ${ }^{[15]}$ 的研究表明悬浮物浓度升高会降低大口黑鲇 (Micropterus salmoides) 对猎物的捕食. Meager 等 ${ }^{[16]}$ 的研究表明, 靠视觉捕食的大西洋䲕鱼 (Gadus morhua) 可通过化学感应捕食以弥补高浓度悬浮物对视觉的 影响,从而削弱悬浮物对捕食的抑制作用.

本研究选取太湖常见的食浮游动物鱼类——餐条 (Hemiculter leucisculus) ${ }^{[17]}$ 作为研究对象. 吕志均等 ${ }^{[18]}$ 的研究表明, 在透明度较高的小型湖泊中, 靠视觉捕食的餐条白天肠含物主要由浮游动物组成 (平均 196 ind. / 尾), 而在夜晚餐条肠含物中浮游动物数量极少 (平均 0.2 ind. /尾), 说明水中的光强能影响餐条 的捕食. 本研究通过模拟不同再悬浮条件, 探究沉积物再悬浮对食浮游动物鱼类捕食浮游动物的影响, 为经 典的生物操纵方法是否适用于亚热带浅水湖泊提供理论依据.

\section{1 材料与方法}

\section{1 实验材料}

取太湖表层底泥, 晾干后过 $0.6 \mathrm{~cm}$ 孔径双层钢篮以去除植物碎屑及螺类等. 将处理过的底泥装人 $950 \mathrm{~L}$ (上底直径 $1.2 \mathrm{~m}$ 、下底直径 $1 \mathrm{~m}$ 、高 $1.2 \mathrm{~m}$ ) 玻璃钢桶中, 装人底泥厚度为 $10 \mathrm{~cm}$. 通过小型造流百 (分别为 7 和 $10 \mathrm{~W})$ 的动力作用分别模拟 2 种不同的悬浮条件 ${ }^{[6]}$. 实验用餐条体长 $10 \mathrm{~cm}$ 左右, 于太湖中捕获. 实验用水 为太湖原水.

\section{2 实验各处理设置}

在桶中加人湖水, 稳定 $1 \mathrm{~d}$ 后安装小型造流洜进行再悬浮强度调试. 实验共设 6 个处理, 处理 1 : 强再悬 浮 + 餐条; 处理 2 : 强再悬浮; 处理 3 : 弱再悬浮 + 餐条; 处理 4 : 弱再悬浮; 处理 5 : 无再悬浮 (不安装造 流原 $)+$ 餐条; 处理 6 : 无再悬浮,每个处理设置 3 个重复. 实验于 2013 年 10 月 $14-29$ 日进行.

\section{3 采样和分析方法}

现场用水质分析仪 YSI 6600 测定水温、pH 和溶解氧等指标. 用 $5 \mathrm{~L}$ 有机玻璃柱状采水器分别采取上、下 层水样各一次, 混合后分析指标. 指标包括总悬浮质浓度 (TSS)、总氮 (TN)、总磷 (TP) 和叶绿素 a 浓度 (Chl. a), 测定方法参照《湖泊富营养化调查规范》 ${ }^{[19]}$; 同时, 取 $5 \mathrm{~L}$ 混合水样用 $25^{\#}$ 浮游生物网过滤, 用蒸馏 水冲刷篮网上浮游动物并定容至 $50 \mathrm{ml}$, 用 $0.5 \mathrm{ml}$ 福尔马林溶液固定后镜检. 采样分别在实验开始和结束时 进行, 采样时间为上午 $8: 00-9: 00$.

\section{4 样品鉴定及数据处理方法}

浮游动物鉴定参照文献 [20-22]. 各处理间差异显著性采用 SPSS 19.0 软件进行方差分析以及 Spearman 分析.

\section{2 结果}

\section{1 实验期间理化因子的变化}

实验开始和结束时的水温平均值分别为 16.6 和 $15.7^{\circ} \mathrm{C}, \mathrm{pH}$ 分别为 8.08 和 8.68 , 溶解氧浓度分别为 7.38 和 $8.10 \mathrm{mg} / \mathrm{L}$, TN 浓度分别为 1.25 和 $1.73 \mathrm{mg} / \mathrm{L}$, TP 浓度分别为 0.061 和 $0.078 \mathrm{mg} / \mathrm{L}$. 各处理间溶解 氧、TN 和 TP 浓度均随再悬浮强度的减弱而减小. 
实验结束时不同再悬浮处理间 TSS 浓度差异极显著 $(P<0.01)$,相同再悬浮条件下有、无餐条处理之间 的 TSS 浓度差异不显著 $(P>0.05)$. 不同再悬浮处理之间 Chl. a 浓度差异极显著 $(P<0.01)$, 而相同再悬浮 条件下, 有、无餐条处理之间 Chl. a 浓度差异均不显著 $(P>0.05)$ (图 1). Chl. a 与 TSS 浓度变化趋势一致 ( 图 1 ).
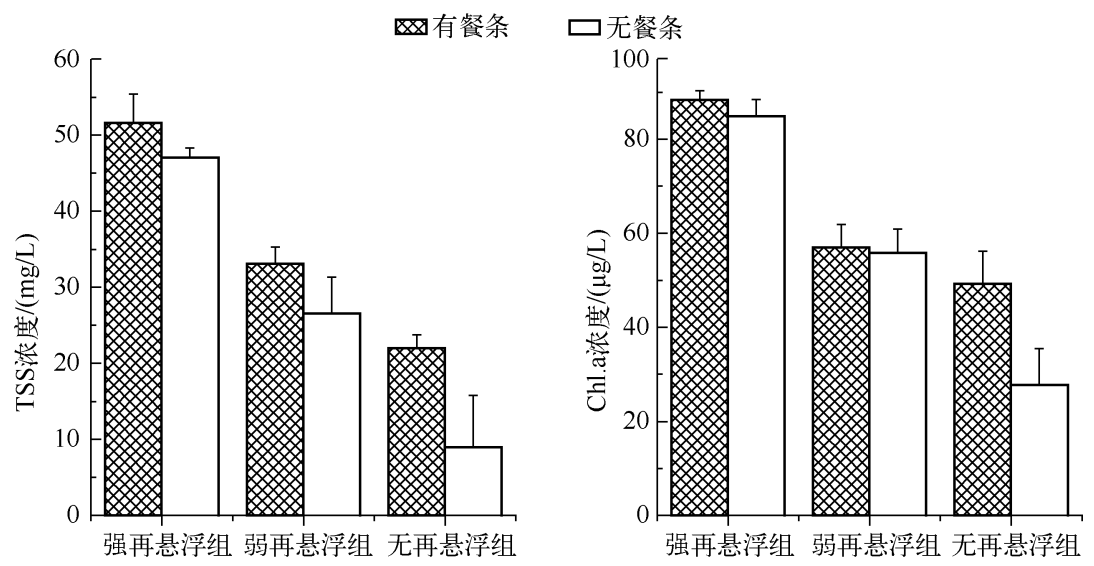

图 1 实验结束时不同处理条件下 TSS 和 Chl. a 浓度

Fig. 1 Concentrations of total suspended solids and chlorophyll-a concentrations in different treatments at the end of the experiment

\section{2 浮游动物群落结构变化}

实验结束时鉴定到的浮游动物种类如表 1 所示.

实验开始时, 各处理之间浮游动物群落结构的 组成和丰度差异不显著 $(P>0.05)$. 实验结束时, 强 再悬浮条件下, 有餐条处理组的枝角类和桡足类丰 度大于无餐条处理组, 轮虫丰度则为无餐条处理组 大于有餐条处理组. 在弱再悬浮组条件下, 有餐条处 理组中枝角类丰度小于无餐条处理组, 而桡足类则 相反, 轮虫丰度无显著差异 $(P>0.05)$. 无再悬浮条 件下, 枝角类、桡足类和轮虫丰度均为有餐条处理组 小于无餐条处理组 (图 2). 方差分析表明, 轮虫丰度 仅在无再悬浮条件下, 有、无餐条处理差异显著 $(P=$ $0.044)$. 桡足类在有、无餐条处理条件下无显著性差 异 $(P>0.05)$. 枝角类在弱再悬浮 $(P=0.010)$ 和无 再悬浮 $(P=0.023)$ 条件下, 有、无餐条处理间均存在 显著差异.

实验结束时,在有餐条处理组中, 大型枝角类短钝溞只在弱再悬浮条件下出现并成为枝角类的优势种. 在无餐条的处理组中, 短钝溞生物量随再悬浮强度减弱而增加. 在强再悬浮条件下以及无再悬浮 + 餐条处 理组中, 小型枝角类角突网纹溞和盘肠溞为优势种. 无论有、无餐条条件下,桡足类优势种均为中剑水蚤,其 生物量均随再悬浮强度减弱而增加 (图 3). 方差分析 (图 3) 表明, 短钝溞生物量在弱再悬浮和无再悬浮条件 下有、无餐条处理间均呈现出极显著差异 $(P<0.01)$; 角突网纹溞 $(P=0.018)$ 和中剑水蚤 $(P=0.023)$ 生物 量仅在无再悬浮条件下有、无餐条处理间呈显著差异;盘肠溞生物量在各悬浮条件下有、无餐条处理间均无 显著差异 $(P>0.05)$.
表 1 实验结束时浮游动物群落结构

Tab. 1 Zooplankton community structures at the end of the experiment

\begin{tabular}{|c|c|}
\hline 类群 & 种类 \\
\hline \multirow[t]{4}{*}{ 枝角类 } & 短针溞 (Daphnia obtusa) * \\
\hline & 盘肠溞( Chydorus sp. ) * \\
\hline & 角突网纹溞 (Ceriodaphnia cornuta) * \\
\hline & 同形溞( Daphnia similis) \\
\hline \multirow[t]{3}{*}{ 桡足类 } & 中剑水蚤 (Mesocyclops sp. ) * \\
\hline & 真剑水蚤 (Eucyclops sp. ) \\
\hline & 无节幼体 (Nauplius) \\
\hline \multirow[t]{3}{*}{ 轮虫 } & 臂尾轮虫 (Brachionus spp. ) * \\
\hline & 晶囊轮虫 (Asplanchnopus spp. ) \\
\hline & 龟甲轮虫 (Keratella spp.) \\
\hline
\end{tabular}

*表示优势种. 

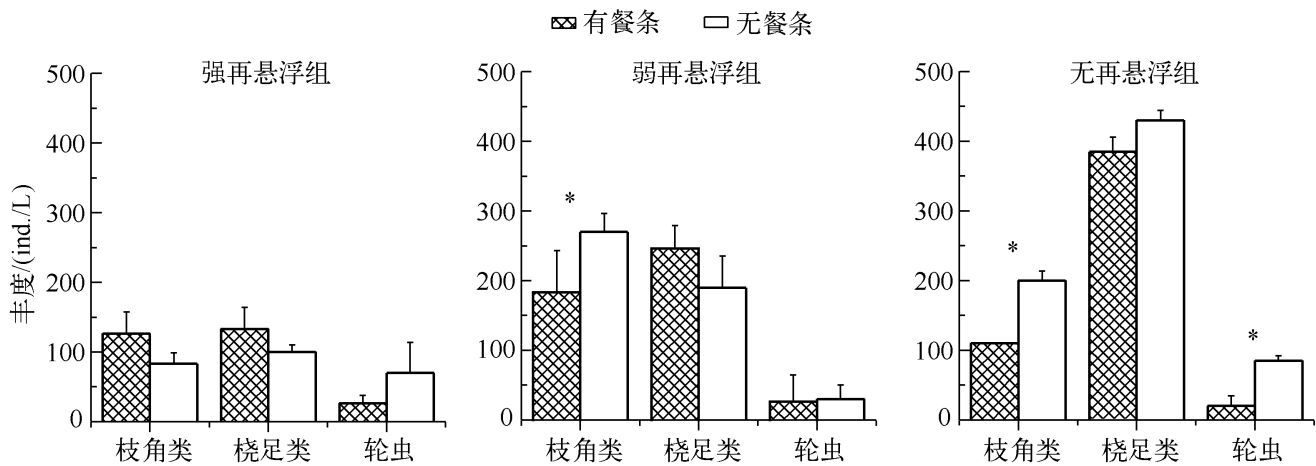

图 2 实验结束时不同处理组浮游动物的丰度 $(*$ 表示有、无餐条处理之间的差异显著, $P<0.05$;

**表示有、无餐条处理之间的差异极显著, $P<0.01$;下同)

Fig. 2 Zooplankton abundance in different treatments at the end of the experiment
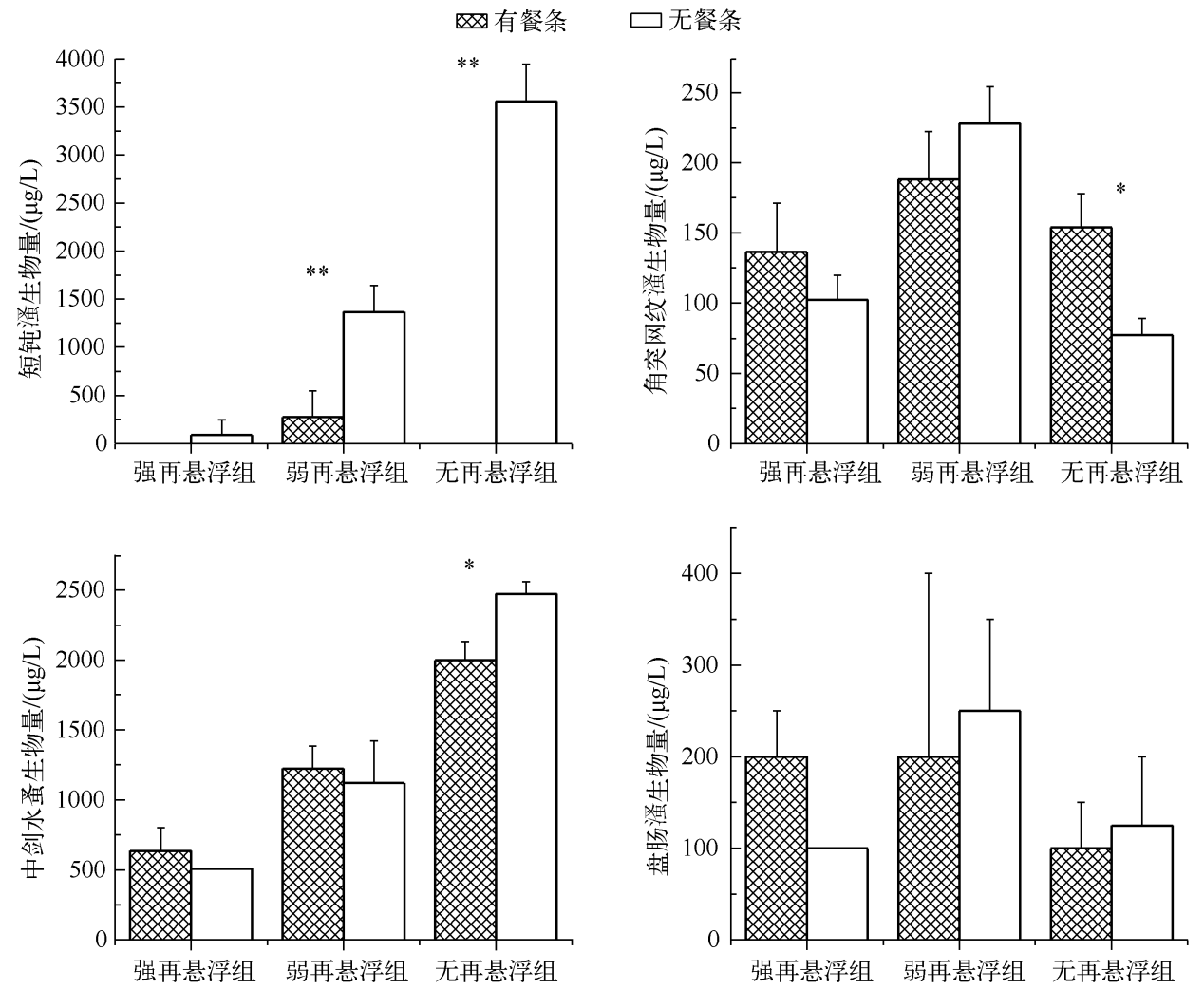

图 3 实验结束时不同处理组浮游动物优势种生物量

Fig. 3 Biomass of dominant zooplankton in different treatments at the end of the experiment

\section{3 讨论}

本研究结果表明,不同的再悬浮强度对浮游动物群落及食浮游动物鱼类捕食浮游动物均有影响. 实验 结束时, 有餐条处理组强、弱、无再悬浮组 TSS 浓度分别为 $51.60 、 33.11 、 22.00 \mathrm{mg} / \mathrm{L}$, 而无餐条处理组 TSS 浓度分别为 $47.07 、 25.56 、 8.95 \mathrm{mg} / \mathrm{L}$, 这 3 个值分别与太湖的湖心区、梅梁湾和五里湖的 $\operatorname{TSS}$ 浓度相近 $^{[23]}$. 
一般而言, 随着 TSS 浓度的升高, 浮游动物有向小型化转变的趋势 ${ }^{[6]}$. 本研究在强再悬浮条件下, 浮游动物 的优势类群为小型枝角类 (角突网纹溞和盘肠溞)、桡足类和轮虫. 对于大型枝角类一一溞来说, 过高的 TSS 浓度能降低其对食物的摄食和吸收, 并且使浮游动物体重增加, 从而使得呼吸作用所需要的能量增加, 直接 或间接降低它们的怀卵率和存活率 ${ }^{[24-27]}$. 桡足类运动速度较快, 能够主动摄食, 因此能降低悬浮物对它的影 响 ${ }^{[28]}$. 在强再悬浮条件下, 中剑水蚤生物量虽然保持较高的水平, 但低于弱再悬浮和无再悬浮条件下的生物 量, 这说明再悬浮强度对桡足类有一定的负面影响. 轮虫与桡足类类似, 也能选择性地取食, 因此能够更好 地适应高浓度悬浮物条件 ${ }^{[6,8]}$. 在无再悬浮条件下, 有餐条的处理组中浮游动物以小型枝角类、桡足类和轮 虫为主, 而未发现大型枝角类溞. 在湖泊生态系统中, 鱼类捕食是决定浮游动物群落结构和数量的关键因 子 ${ }^{[29]}$. 餐条作为食浮游动物鱼类, 其存在抑制了大型浮游动物溞的生存. 餐条食性分析也表明, 其在水体中 优先捕食溞, 而少量捕食中剑水虫、角突网纹掻和轮虫 ${ }^{[30]}$. 因此, 在无再悬浮 + 餐条处理组中未发现溞, 而中 剑水蚤和小型枝角类的数量较多. 在无餐条处理组中, 由于没有鱼类的捕食, 大型枝角类短钝溞的生物量显 著高于小型枝角类角突网纹溞和盘肠溞的生物量.

Redding 等 $^{[14]}$ 和 Shoup 等 ${ }^{[15]}$ 的研究表明, 沉积物再悬浮能够破坏鱼类的鳃结构, 从而干扰其捕食. 吕志 均等 ${ }^{[18]}$ 研究发现, 靠视觉捕食的餐条白天肠含物主要由浮游动物组成, 而夜晚以碎屑为主, 因此悬浮物能通 过改变水体的光强来干扰鱼类的捕食. 在本研究 3 个再悬浮 + 餐条处理组中, 只有弱再悬浮 + 餐条处理组 出现了大型枝角类溞. 通过上述分析, 强再悬浮 + 餐条处理组中, 溞的缺失主要是由于强再悬浮的干扰所 致; 而无再悬浮 + 餐条处理中, 溞的缺失主要是鱼类的捕食所致. 在弱再悬浮 (无餐条) 处理组中, 大型枝角 类短钝溞的生物量显著高于强再悬浮 (无餐条) 处理组, 但显著低于无再悬浮 (无餐条) 处理组, 说明大型枝 角类短钝溞已能够适应本研究中弱再悬浮 ( TSS 浓度为 $30 \sim 35 \mathrm{mg} / \mathrm{L}$ ) 条件. 在弱再悬浮处理中, 有餐条条件 下短钝掻的生物量低于无餐条条件, 说明餐条能够捕食短钝泽, 但由于悬浮物本身的干扰作用以及对光强的 影响, 制约了餐条对短钝溞的控制. Chen 等 ${ }^{[31]}$ 通过对无锡五里湖生态修复前后 12 年的数据分析表明, 生态 修复后可能由于底层鱼类 (鲤、鲫) 的干扰, 使得 TSS 浓度由修复前的 $20 \sim 30 \mathrm{mg} / \mathrm{L}$ 增加到 $30 \sim 40 \mathrm{mg} / \mathrm{L}$, 而 湖中尚存大量食浮游动物鱼类, 如湖鲚 (Coilia ectenes taihuensis)、鲢 (Hypophthalmichthys molitrix)、鳙 (Aristichthys nobilis)、餐条等 ${ }^{[32]}$. 修复后一些大型枝角类溞和透明薄皮溞 (Leptodora kindti) 相继出现, 说明一定浓 度的 TSS 能干扰食浮游动物鱼类对浮游动物的捕食. 因此, 野外数据和本研究模拟实验都表明, 适当的 TSS 浓度既能干扰食浮游动物鱼类对大型浮游动物的捕食, 又不影响大型浮游动物的生存, 使得提高浮游动物 对藻类的牧食强度成为可能.

致谢: 何虎、王文侠、魏小飞、谷娇在实验过程中给予了很大帮助,在此表示衰心感谢.

\section{4 参考文献}

[ 1 ] Jeppesen E, Meerhoff M, Jacobsen BA et al. Restoration of shallow lakes by nutrient control and biomanipulation-the successful strategy varies with lake size and climate. Hydrobiologia, 2007, 581 : 269-285.

[ 2 ] Søndergaard M, Jeppesen E, Lauridsen T et al. Lake restoration: successes, failures and long-term effects. Journal of Applied Ecology, 2007, 44 : 1095-1105.

[ 3 ] Roozen FCJM, Lürling M, Vlek H et al. Resuspension of algal cells by benthivorous fish boosts phytoplankton biomass and alters community structure in shallow lakes. Freshwater Biology, 2007, 52 : 977-987.

[ 4 ] Ogilvie BG, Mitchell SF. Does sediment resuspension have persistent effects on phytoplankton? Experimental studies in three shallow lakes. Freshwater Biology, 1998, 40: 51-63.

[ 5 ] Schallenberg M, Burns CW. Effects of sediments resuspension on phytoplankton production: teasing apart the influences of light, nutrients and algal entrainment. Freshwater Biology, 2004, 49 : 143-159.

[ 6 ] 蒋伟伟,刘正文, 郭 亮等. 沉积物再悬浮对浮游动物群落结构影响的模拟实验. 湖泊科学,2010,22(4): 557-562. DOI 10. 18307/2010.0412.

[ 7 ] Arruda JA, Marzolf GR, Faulk RT. The role of suspended sediments in the nutrition of zooplankton in turbid reservoirs. Ecology, 1983, 64(5) : 1225-1235.

[ 8 ] Kirk KL, Gilbert JJ. Suspended clay and the population dynamics of planktonic rotifers and cladocerans. Ecology, 1990, 
$71(5): 1741-1755$.

[ 9 ] Song XL, Liu ZW, Yang GJ et al. Effects of resuspension and eutrophication level on summer phytoplankton dynamics in two hypertrophic areas of Lake Taihu, China. Aquatic Ecology, 2010, 44: 41-54.

[10] Ghadouani A, Pinel-Alloul B, Prepas EE. Efforts of experimentally induced cyanobacterial blooms on crustacean zooplankton communities. Freshwater Biology, 2003, 48: 363-381.

[11] Nurminen L, Pekcan-Hekim Z, Repka S et al. Effect of prey type and inorganic turbidity on littoral predator-prey interactions in a shallow lake: an experimental approach. Hydrobiologia, 2010, 646: 209-214.

[12] Bonner TH, Wilde GR. Effects of turbidity on prey consumption by prairie steam fishes. Transactions of the American Fisheries Society, 2010, 131 : 1203-1208.

[13] Granqvist M, Mattila J. The effects of turbidity and light intensity on the consumption of mysids by juvenile perch(Perca fluviatilis L. ). Hydrobiologia, 2001, 514: 93-101.

[14] Redding JM, Schreck CB, Everest FH. Physiological effects on Coho Salmon and steelhead of exposure to suspended solids. Transactions of the American Fisheries Society, 1987, 116: 737-744.

[15] Shoup DE, Wahl DH. The effects of turbidity on prey selection by piscivorous largemouth bass. Transactions of the American Fisheries Society, 2009, 138: 1018-1027.

[16] Meager JJ, Solbakken T, Utne-Palm AC et al. Effects of turbidity on the reactive distance, search time, and foraging success of juvenile Atlantic cod(Gadus morhua). Canadian Journal of Fish and Aquatic Science, 2005, 62 : 1978-1984.

[17］毛志刚,谷孝鸿, 曾庆飞等. 太湖鱼类群落多结构及多样性. 生态学杂志,2011,30(12):2836-2842.

[18］吕志均, 戴 䂀, 孙 颖等. 紫霞湖浮游甲壳动物昼夜垂直迁移及其影响因子分析. 水生态学杂志, 2011,32(3): $43-47$.

[19］金相灿, 屠清瑛. 湖泊富营养化调查规范. 北京:中国环境科学出版社,1990.

[20] 蒋卒治, 堵南山. 中国动物志: 淡水枝角类. 北京:科学出版社, 1979 .

[21] 沈嘉瑞,戴爱云,张崇洲. 中国动物志:淡水桡足类. 北京:科学出版社,1979.

[22] 王家楫. 中国淡水轮虫志. 北京:科学出版社, 1961 .

[23] 张运林,秦伯强,陈伟民等. 太湖水体中悬浮物研究. 长江流域资源与环境,2004,13(3):266-271.

[24 A Arruda JA, Marzolf GR, Faulk RT. The role of suspended sediments in the nutrition of zooplankton in turbid reservoirs. Ecology, 1983, 64(5) : 1225-1235.

[25] Zurek R. Effect of suspended materials on zooplankton. 2. Laboratory investigations of Daphnia hyalina Leydig. Acta Hydrobiologia, 1983, 24 : 233-251.

[26] Löffle H. Neusiedlersee: the limnology of a shallow lake in central Europe: Monographiae Biologicae 37. In: Illies J ed. The zooplankton of the open lake. The Hague-Boston-London: Dr. W. Junk Publ, 1979: 281-335.

[27] McCabe GD, ÓBrien WJ. The effects of suspended silt on feeding and reproduction of Daphnia pulex. American Midland Naturalist, 1983,110 : 324-337.

[28 ] Thorpe JH, Covich AP. Ecology and classication of north American freshwater invertebrates. In: Williamson CE, Reid JW eds. Copepoda. San Diego CA: Academic Press, 2001: 915-954.

[29] Gliwicz ZM, Pijanowska J. The role of predation in zooplankton succession. In: Somme U ed. Plankton ecology. New York: Springer-Verlag, 1989: 253-296.

[30］叶佳林. 太湖梅梁湾沿岸带鱼类组成和摄食生态研究 [学位论文].武汉:华中农业大学,2006.

[31] Chen FZ, Shu TT, Jeppesen E et al. Restoration of a subtropical eutrophic shallow lake in China: effects on nutrient concentrations and biological communities. Hydrobiologia, 2013, 718: 59-71.

[32] 张宪中, 胡海彦, 曹晓东等. 五里湖鱼类资源群落结构及生物多样性的时空分析. 大连海洋大学学报, 2010,25(4): 314-319. 